bination of constitutional and surrounding conditions, or entering as a specific germ through a wound, it is a poison having a special affinity for certain structures, and to these it passes at once. The structures are here assumed to be the synovial membranes. In what this affinity or altractiveness consists we are as yet ignorant, and can but illustrate it by what takes place in other diseases, such as the cholera poison, and the scarlatinal poison, or the producer of tetanus; whether it is a something evolved within the system, or introduced from without, it goes at once to certain nerve-tissues, and in them creates changes which set up other morbid action. Many drugs illustrate the same fact. Strychnia when swallowed, or when subcutaneously injected, has but one form of action, like the poison of tetanus, -it affects nerve-tissue only. The strong analogy between pyæmia at the commencement, and acute rhermatism, has often been observed. In both there are the rigors, the fever, the rapid pulse, the profuse sweating, but above all there is the pain and swelling of one or more joints. It seems probable that in both an entity has entered the system whose bahitat is the joints. We cannot tell in what consists the difference of the two poisons, so that the one as a rule eventuates in recovery, and the other in a train of pathological changes whose termination is death. In both cases the tissue first affected is the synovial membrane. The abnormal action induced in it leads to an increased secretion of synovia, probably unaltered in its character and constituents in rheumatism, but of an abnormal kind in pyæmia. In the case of the synovial fluids there is in most joints a limit to its quantity; so tightly is the synovial sac compressed by surrounding tissues which are not yielding that an amount of tension quickly ensues, which leads to a forced absorption of some of the effused fluid, and then ensues in acute rheumatism, and probably as a necessary sequence, an extension of the disease to other synovial sacs, and often to the pericardium, a serous membrane, but closely allied in its nature to a synovial membrane. This augmented synovial fluid is in rheumatism a bland and innocuous fluid-a mere increase of the natural secretion; but in pyæmia it is in a decomposing state, developing rapidly germs of a lower organisation; and when such a fluid bas been absorbed, and in its course reaches the minute capillaries of the lungs, some of its morbid cells coagulate the fibrine of the blood there, and become arrested, and thus are formed the nuclei with which the lungs are studded, around which more fibrine is deposited, and the pathological changes follow described by Virchow and others.

In conclusion, the author states that in limiting the paper to a consideration of the fact of the synovial membranes being the tissues first affected in many forms of pyæmia, he has not lost sight of the probability of many otber phases of pyæmia having their beginnings in one or other of the serous membranes, these two membranes being so closely allied in their microscopic characters.

Mr. HULKE failed to understand the theory of the author as to the re-entrance of fluid into the joints, and hoped to have heard some suggestion explanatory of the source of pus found in the joints, and bow bacteria, \&c., originate under such circumstances. He also averred his belief that many cases of præmia occur without any affections of joints.

Mr. Woon failed to understand how joints can, as it were, manufacture a poison without some predisposition on the part of the fluids of the body, and that then the joints only should be affected.

Mr. HamiLton, in replv, regretted that he bad not been sufficiently understood. He believed that the fluid spread from one joint to another, on account of the pressure exercised on the synovial membrane, which forced the fluid into the circulation. His observations as to the presence of living organisms in the fiud were made irrespective of the results recorded by Dr. Burdon Sanderson. He had never any case of pjæmia witbout some stiffness, if not positively some acute affection, of the joints.

ON SOME FURTHER ATTEMPTS TO CURE LARGE INTERNAL ANEURISMS.

BY WILLIAM MURRAY, M.D. (NEWCASTLE.)

(Communicated by Dr. Symes Thомpson.)

This is an account of some attempts to cure internal aneurisms by the insertion of foreign bodies with a view to the formation of clots in the interior of the sac. Some of these bodies were introduced and again withdrawn (needles), and their introduction was repeated at intervals with manifest thickening of the aneurismal walls on each occasion; others were of a material the presence of which would be as harmless as possible (carbolised catgut), and they were used because of the hope that their absorption might take place after the work of coagulation was completed. Lastly, wire was tried, as in Mr Moore's case, and with more favourable results as regards the preservation of the patient's life, he having lain more than three weeks with twenty-four feet of wire in him. Although none of these efforts were actually successful in curing the patient, they mark out a line of practice which may some day be perfectly safe and successfnl.

Mr. Holmes thought Dr. Murray's paper useful as tending to show the barmlessness of such an operation, and that surgeons might meddle with aneurisms to a mnch greater extent than was generally imagined. After remarking that the introduction of carbolised catgut was manifestly useless (because it would soften rapidly) he said that Mr. Moore's case (the preparation is now in the Middlesex Hospital) showed markedly the dangers of the operation, and it was evident that wire or any other material of sufficient hardness to help coagulation when introduced in to the sac, must, by rubbing against the walls of the latter, tend to set up a dangerous degree of inflammation.

Dr. ANDREW demurred to the wisdom of any surgical interference, inasmuch as external ruptures of the sac are extremely rare, and any modes of procedure such as those described would tend to produce a clot around the strongest instead of the weakest portion of the sac.

The last meeting of this session will be held on the 28 th inst.

\section{CLINICAL SOCIETY OF LONDON.}

FRIDAY, MAY 10TH, 1872.

Sir William Gull, Bart., M.D., F.R.S., in the Chair.

Dr. BunNer Yeo read a paper on a case of Paralysis of the Senses of Taste and Smell following Concussion of the Brain. The patient, a man aged fifty, came to King's College Hospital on January 11th, baving four montbs before been thrown out of a cart. He was admitted into St. Thomas's Hospital in a semi-conscious state, under the care of $\mathrm{Mr}$. Croft. A slight contusion was discovered on the back of the head, but there was no fractured bone. He vomited, was delirious and insensible during the night, and in the morning complained of much pain in the head, which was relieved by aperients. He soon recovered, and, feeling well, discharged himself after he had been in the hospital about a week. He found, however, that he could neither taste nor smell. This condition had persisted ever since the accident. He had suffered also a good deal from giddiness, and from severe pains on the right side of the head. He had been a perfectly healthy man, and quite free from any syphilitic taint. The patient's statements were thoroughly tested. Salt, sugar, solution of quinine, dilnte acids, tincture of assafœtida, were applied to the tip, the centre, and the back part of the tongue, to the under as well as the upper surface, but were not recognised in any degree. The tongue, however, was sensitive to the sligbtest touch, and its movements were perfect. The sense of smell, similarly tested, was found to be absent. The patient was ordered five grains of iodide of potassium three times a day. The first two doses produced such violent symptoms of iodism that it could not be continued. The dose was therefore reduced to two grains; and as this also acted in the same way, only one grain three times a day was given. This dose, at first, produced iodism, and afterwards purged him; he, however, continued to take it. In a few days the sense of taste began to return. On January 25 th he conld taste solution of quinine as bitter, and tincture of assafotida as bitter; but he could detect no odour in the latter. $\mathrm{H}_{\theta}$ could distinguish salt from sugar, but not tea from coffee. The sense of taste continued to return rapidly. On Feb. 11 th he was able to taste the flavcur of meat for the first time for twenty weeks. He could also now perceive strong odours, but smell came back less rapidly and less perfectly than taste. In connexion with the return of smell, he 